Type of article: Original Research Manuscript

\title{
Title: Clinical advantage of attaining index-based remission prior to composite remission in treating rheumatoid arthritis
}

Running title: CDAI/SDAI remission first, then Boolean

Authors: Ichiro Yoshii ${ }^{1}$, Tatsumi Chijiwa ${ }^{2}$, and Naoya Sawada ${ }^{3}$

Affiliations:

1) Department of Rheumatology and Musculoskeletal Medicine, Yoshii Hospital, 6-7-5 Nakamura-Ohashidori, Shimanto City, 787-0033 Kochi Prefecture, Japan

2) Department of Rheumatology, Kochi Memorial Hospital, 4-13 Shiromi-cho, Kochi, 780-0824 Kochi, Japan

3) Department of Rheumatology, Dohgo Onsen Hospital, 21-21 Himetsuka-Otsu, Matsuyama, 790-0858 Ehime Prefecture, Japan

\begin{abstract}
ABSTARACT
Background and objectives: The clinical advantage of targeting index-based remission prior to Boolean remission was evaluated retrospectively. Materials and Methods: A total of 578 patients with rheumatoid arthritis (RA), who were treated for more than three years, were recruited. Patients who were treated to targeted index-based remission and composite measure remission criteria such as Boolean remission from the first consultation were divided according to the turn of attaining Boolean remission and indexbased remission: G-IBR, a group that matched index-based remission at the same time Boolean remission is attained or earlier; G-BR_IF, a group that attained Boolean remission followed by index-based remission or failed; G-IR BF, a group that could not attain Boolean remission despite attaining index-based remission; G-BothF, a group that failed to attain either Boolean remission or index-based remission. Background factors were statistically compared among groups. The Boolean remission rate in patients who attained index-based remission (BRR) and the rate of failure to attain index-based remission in patients who failed to attain Boolean remission (BFR) were statistically evaluated. Results: Groups made of 225, 231, and 482 in G-IBR; 160, 154, and 8 in GBR IF; 18, 18, and 75 in G-IR BF; and 175, 175, and 13 in G-BothF when indexing the clinical disease activity index (CDAI), simplified disease activity index (SDAI), and 28joints disease activity score with C-reactive protein (DAS28-CRP), respectively. Disease activity indices' scores after Boolean remission demonstrated significantly higher in the G-BR_IF group than in the G-IBR group. BRR was $92.6 \%, 92.8 \%$, and $86.5 \%$, while BFR was $71.3 \%, 71.3 \%$, and $13.8 \%$ when indexing CDAI, SDAI, and DAS28-CRP, respectively. Conclusions: Targeting CDAI and SDAI remission prior to Boolean remission contributes to a stable clinical course.
\end{abstract}

Keywords: rheumatoid arthritis; remission; ADL; therapeutic index 


\section{Main document Introduction}

Since the treat to target strategy (T2T) has been advocated worldwide for rheumatoid arthritis (RA) therapy [1], there is a broad consensus that clinical remission should be the initial target to achieve [2]. The state of clinical remission is generally defined as the absence of signs and symptoms of inflammation [1]. Various definitions of remission exist; presently, most popular index-based criteria like 28-joint disease activity score using C-reactive protein (DAS28-CRP) [3], clinical disease activity index (CDAI), simplified disease activity index (SDAI) [4], or composite measures such as Boolean evaluation [5] were widely indexed for the judgment of clinical remission in disease activity. In these, the Boolean remission is defined as all of the four parameters are controlled, such as tenderness joint count $(\mathrm{TJC}) \leq 1$, swollen joint count $(\mathrm{SJC}) \leq 1$, patient's global assessment (PGA) $\leq 1$, and CRP $\leq 1 \mathrm{mg} / \mathrm{dl}$. These criteria may be most stringent in the four criteria, and it is a broad consensus that RA treatment is successful when the disease activity is in Boolean remission because continuous Boolean remission guarantees good radiographic and functional outcomes after at least a 2-year follow-up $[5]$.

On the other hand, controversial opinions also evoked that the Boolean remission is too stringent [6]. The reason for the opinion is that PGA $\leq 1$ does not represent an objective disease activity status, and one report suggested that PGA $\leq 2$ may also be available for the evaluation of remission [7]. Index-based remission criteria such as CDAI, SDAI, and DAS28-CRP maintain PGA $\leq 2$ as long as the summarized number of components fulfills the limited number of remission criteria. Namely, CDAI remission is defined as a sum total of TJC, SJC, PGA, and evaluators' global assessment (EGA) $\leq 2.8_{2}$ whereas SDAI remission is defined as a sum total of TJC, SJC, PGA, EGA, and CRP $\leq$ 3.3, and DAS28-CRP is defined as a calculated number of formula with TJC, SJC, PGA, and CRP $<2.6$. These criteria may play the same role for the stringent index for the remission that can guarantee a stable clinical course after attaining remission except for DAS28-CRP remission [8].

We have investigated patients' time course of disease activity and evaluated how the clinical course in the patient with RA should be targeted in accordance with T2T.

\section{Methods}

We have been treating patients with RA in accordance with T2T since August 2010. For these patients, TJC, SJC, PGA, EGA, CRP, the Health Assessment Questionnaire Disability Index (HAQ-DI) [9], a pain scale with visual analog scale (PSVAS) [10], and the EuroQOL-5 dimensions (EQ-5D) [11] were monitored every other chance of consultation from the first consultation (baseline). Roentgenographic evaluation with the Sharp/van der Heijde Score (SHS) [12] from the X-ray pictures of bilateral hand and foot was taken every other year from baseline. We set a clinical target for patients from baseline and defined remission as CDAI $\leq 2.8$. Treatment for RA targets CDAI remission, which is one of index-based remission criteria within six months after baseline.

A total of 578 patients with RA who were treated at our institute using T2T [1,2] for more than three consecutive years were recruited. The patients were divided into groups according to their attainment of the Boolean remission and attainment of the targeted index-based remission criteria: G-IBR, a patient group who attained index-based 
remission at the same time as attaining Boolean remission or earlier; G-BR IF, a patient group that attained Boolean remission followed by index-based remission or failed to attain index-based remission; G-IR BF, a patient group that could not attain Boolean remission despite attaining index-based remission; G-BothF, a patient group that failed to attain either Boolean remission or index-based remission. Background factors such as sex distribution (female percentage), age, disease duration, anti-cyclic citrullinated polypeptide-antibody (ACPA)-positive rate, rheumatoid factor (RF)-positive rate, and composite measures configures and index-based criteria at baseline were compared with each of the other groups using the one-way ANOVA test with Bonferroni correction. Other clinical indices such as HAQ-DI, pain score measured with the visual analog scale (PS-VAS), and the quality of life score (QOLS) [13], which was calculated from the EuroQOL 5 dimensions (EQ-5D) at baseline were also compared using the one-way ANOVA test with Bonferroni correction.

Average values of these clinical components upon remission criterion from baseline to the first Boolean remission (before remission) and the average values of these parameters from the acquisition to last observation (after remission) in the G-IBR and the G-BR_IF groups were also compared using the Mann-Whitney U test.

Methotrexate (MTX) administration rate and mean dosage of MTX, biologic or targeted synthetic disease-modifying anti-rheumatic drug (b-/ts-DMARD) administration rate, and glucocorticoid steroid (GCS) administration rate and mean dosage of GCS before and after remission in the two groups were also compared using the Mann-Whitney U test.

Boolean remission rate per total number of consultation was compared to the index-based remission rate, and failure to attain index-based remission rate in the patients who did not attain Boolean remission was evaluated.

These procedures are carried out with regard to the variant index-based remission criterions with CDAI, SDAI, or DAS28-CRP. Subject distributions with the three criterions were compared using chi square test.

All statistical analyses were performed using StatPlus:mac ${ }^{\circledR}$ (AnalystSoft, Inc., Walnut, CA, USA).

\section{Results}

A total of 578 subjects were included in the study. According to the three indexbased criterions, the CDAI criteria configured $225,160,18$, and 175 in the G-IBR, the GBR IF, the G-IR BF, and the G-BothF group, respectively, whereas the SDAI criteria configured $231,154,18$, and 175 . There was no significant difference between the two variant criterions. On the other hand, the DAS28-CRP criteria configured 482, 8, 75, and 13 , in the G-IBR, the G-BR IF, the G-IR BF, and the G-BothF group, respectively. There was no case with missing data. Subject distribution with the DAS28-CRP criteria was significantly different compared with the other two variant criterions. Demographic and clinical parameters at baseline of the groups for each index-based remission variant are demonstrated in Table 1. Sex distribution, ACPA positive rate, RF positive rate, CDAI, SDAI, DAS28-CRP, and PS-VAS at baseline demonstrated no significant difference between any pairs of the four groups, while the average age in the G-IR BF group was significantly greater than that in the G-IBR and G-BR_IF groups with regard to the CDAI and SDAI. Disease duration and the HAQ score at baseline were significantly greater in the $\underline{\mathrm{G}-\mathrm{B} \text { BothF }}$ group than in the G-IBR and the $\underline{\mathrm{G}-\mathrm{BR} \text { IF }}$ groups with regard to CDAI and 
SDAI. SHS at baseline in the G-BothF was significantly greater than in the other groups with regard to CDAI and SDAI. QOLS was significantly lower in the G-BothF group than in the G-IBR and the G-BR_IF group. In contrast, there was no significant difference of the parameters at baseline between any of the groups with regard to DAS28-CRP.

Table 1: Demographic characteristics of the groups classified according to each index-based remission criteria, and clinical indices at baseline

\begin{tabular}{|c|c|c|c|}
\hline & CDAI & SDAI & DAS28-CRP \\
\hline Case numbers & $225: 160: 18: 175$ & $231: 154: 18: 175$ & $482: 8: 75: 13$ \\
\hline female percentage $(\%)$ & $75.5: 76.0: 78.3: 80.7$ & $75.3: 76.3: 78.3: 80.7$ & $76.3: 70.0: 81.2: 72.7$ \\
\hline average age (year) & $68.1: 67.6: 78.2: 71.1\left(^{*}\right)$ & $68.4: 67.4: 78.2: 71.1\left(^{*}\right)$ & $70.4: 68.4: 68.8: 57.0$ \\
\hline $\begin{array}{c}\text { Disease duration at baseline } \\
\text { (years) }\end{array}$ & $5.8: 5.5: 8.4: 10.5(* *)$ & $5.7: 5.6: 8.4: 10.5(* *)$ & $6.8: 5.0: 7.0: 13.0$ \\
\hline ACPA positive rate $(\%)$ & $73.3: 68.8: 73.9: 73.2$ & $73.3: 68.9: 73.9: 73.2$ & $73.1: 80.0: 78.4: 83.3$ \\
\hline RF positive rate $(\%)$ & $71.3: 75.0: 76.2: 72.5$ & $71.3: 75.0: 76.2: 72.5$ & $71.3: 70.0: 74.6: 66.7$ \\
\hline CDAI at baseline & $23.1: 23.7: 20.2: 27.8$ & $23.2: 23.5: 20.2: 27.8$ & $20.3: 25.5: 24.2: 18.2$ \\
\hline SDAI at baseline & $24.0: 24.7: 20.9: 29.1$ & $24.1: 24.6: 20.9: 29.1$ & $21.2: 26.4: 25.0: 19.0$ \\
\hline DAS28-CRP at baseline & $4.0: 4.0: 3.7: 4.3$ & $4.0: 4.0: 3.7: 4.3$ & $4.2: 4.5: 4.4: 3.7$ \\
\hline PS-VAS at baseline & $49.6: 52.1: 32.0: 54.9$ & $49.6: 52.2: 32.0: 54.9$ & $49.3: 50.0: 48.0: 65.0$ \\
\hline HAQ score at baseline & $\begin{array}{c}0.621: 0.495: 0.778: 0.929 \\
(* *)\end{array}$ & $\begin{array}{c}0.652: 0.483: 0.778: 0.929 \\
(* *)\end{array}$ & $0.651: 0.475: 0.706: 0.750$ \\
\hline SHS at baseline & $\begin{array}{c}37.1: 51.5: 52.8: 121.5 \\
(* * *)\end{array}$ & $\begin{array}{c}38.4: 47.3: 52.8: 121.5 \\
(* * *)\end{array}$ & $77.9: 49.0: 104.6: 187.0$ \\
\hline QOLS at baseline & $\begin{array}{c}0.826: 0.831: 0.786: 0.729 \\
(\$)\end{array}$ & $\begin{array}{c}0.828: 0.840: 0.786: 0.729 \\
(\$)\end{array}$ & $0.825: 0.895: 0.772: 0.737$ \\
\hline
\end{tabular}

In all columns, numbers were shown in order of G-IBR, G-BR_IF, G-IR BF, and G-BothF separated with colon.

Patient distributions between with the CDAI and the SDAI criterions demonstrated no significant difference, whereas that with DAS28-CRP was significantly difference compared with the other two variant criterions $(p<0.01)$.

Abbreviations: G-IBR, a patient group who attained index-based remission criteria at the same time as attaining Boolean remission or earlier; G-BR_IF, a patient group who attained Boolean remission followed by index-based remission criteria or failed; G-IR_BF, a patient group who could not attain Boolean remission despite attaining index-based remission criteria ; $\underline{\text { G- }}$ BothF, a patient group who failed attaining neither Boolean remission nor index-based remission criteria; ACPA, anti-cyclic citrullinated polypeptide antibodies ; RF, rheumatoid factor ; CDAI, clinical disease activity index ; SDAI, simplified disease activity index ; DAS28-CRP, 28-joints disease activity score with C-reactive protein ; PS-VADS, pain score measured with visual analog scale ; HAQ, Health Assessment Questionnaire Disability Index ; SHS, Sharp/van der Heijde Score ; QOLS, quality of life score calculated from EuroQOL 5 dimensions.

(*): significantly greater in G-IR_BF than in G-IBR and G-BR IF.

(**): significantly greater in G-BothF than in G-IBR and G-BR IF.

$(* * *)$ : significantly greater in G-BothF than in G-IBR, $\underline{\text { G-BR IF }}$ and G-IR_BF.

(\$): significantly smaller in G-BothF than in G-IBR and G-BR IF.

There was no statistical significance with the DAS28-CRP because of number of cases in the G-IR_BF was too small.

The average values of clinical indices from the baseline to the first Boolean remission and after the first Boolean remission are shown in Table 2. CDAI, SDAI, and 
DAS28-CRP demonstrated significant improvement during the time from the first Boolean remission to the last observation than during the time from the baseline to the first Boolean remission with regard to both of CDAI and SDAI. Between the G-IBR and the G-BR IF groups, the HAQ score, SHS, and QOLS demonstrated no significant difference throughout treatment course. PS-VAS was significantly smaller in the G-IBR than in the G-BR IF from the baseline to the first Boolean remission, however, the difference diminished significance after the first Boolean remission.

Table 2: Average values of clinical indices of the groups from baseline to first Boolean remission and after Boolean remission for each variant criterion with CDAI, SDAI, and DAS28-CRP

\begin{tabular}{|c|c|c|c|c|}
\hline & $\underline{\mathrm{CDAI}}$ & $\underline{\text { SDAI }}$ & $\underline{\mathrm{DAS} 28-\mathrm{CRP}}$ & $p$-value \\
\hline Cases & $225 / 160$ & $231 / 154$ & $482 / 8$ & \\
\hline $\begin{array}{c}\text { Time span from } \\
\text { baseline to Boolean } \\
\text { remission }\end{array}$ & $5.4 / 6.8$ & $5.8 / 6.2$ & $\underline{8.1 / 6.0}$ & $\underline{6.7 \times 10^{-2} / 3.4 \times 10^{-1}}$ \\
\hline $\begin{array}{c}\text { CDAI before } \\
\text { Boolean remission }\end{array}$ & $1.52 / 8.08$ & $1.75 / 7.95$ & $\underline{8.23 / 9.94}$ & $\leq 1.0 \times 10^{-12}$ \\
\hline $\begin{array}{c}\text { SDAI before } \\
\text { Boolean remission }\end{array}$ & $2.40 / 8.54$ & $2.67 / 8.48$ & $9.03 / 10.88$ & $\leq 1.0 \times 10^{-12}$ \\
\hline $\begin{array}{l}\text { DAS28-CRP before } \\
\text { Boolean remission }\end{array}$ & $1.77 / 4.71$ & $1.92 / 4.45$ & $\underline{2.32 / 2.79}$ & $\leq 1.0 \times 10^{-12}$ \\
\hline $\begin{array}{l}\text { PS-VAS before } \\
\text { Boolean remission }\end{array}$ & $19.4 / 31.8$ & $20.1 / 29.9$ & $\underline{29.6 / 26.3}$ & $\leq 1.0 \times 10^{-12}$ \\
\hline $\begin{array}{l}\text { HAQ score before } \\
\text { Boolean remission }\end{array}$ & $0.502 / 0.499$ & $0.501 / 0.500$ & $\underline{0.458 / 0.271}$ & $\underline{9.4 \times 10^{-1} / 9.7 \times 10^{-1}}$ \\
\hline $\begin{array}{l}\text { SHS before Boolean } \\
\text { remission }\end{array}$ & $37.1 / 51.5$ & $38.4 / 47.3$ & $\underline{37.5 / 18.5}$ & $\underline{5.0 \times 10^{-2} / 8.2 \times 10^{-2}}$ \\
\hline $\begin{array}{c}\text { QOLS before } \\
\text { Boolean remission }\end{array}$ & $0.830 / 0.829$ & $0.829 / 0.829$ & $\underline{0.7801 / 0.917}$ & $9.9 \times 10^{-1} / 9.9 \times 10^{-2}$ \\
\hline $\begin{array}{l}\text { CDAI score after } \\
\text { Boolean remission }\end{array}$ & $0.46 / 3.50$ & $0.51 / 3.44$ & $2.83 / 4.88$ & $\leq 1.0 \times 10^{-12}$ \\
\hline $\begin{array}{l}\text { SDAI score after } \\
\text { Boolean remission }\end{array}$ & $0.70 / 3.78$ & $0.72 / 3.74$ & $\underline{3.25 / 5.78}$ & $\leq 1.0 \times 10^{-12}$ \\
\hline $\begin{array}{l}\text { DAS28-CRP after } \\
\text { Boolean remission }\end{array}$ & $1.65 / 2.27$ & $1.68 / 2.25$ & $\underline{1.64 / 2.17}$ & $\leq 1.0 \times 10^{-12}$ \\
\hline $\begin{array}{l}\text { PS-VAS after } \\
\text { Boolean remission }\end{array}$ & $19.6 / 22.7$ & $20.0 / 22.6$ & $29.6 / 26.3$ & $\underline{4.2 \times 10^{-1} / 6.3 \times 10^{-1}}$ \\
\hline $\begin{array}{l}\text { HAQ score after } \\
\text { Boolean remission }\end{array}$ & $0.489 / 0.451$ & $0.487 / 0.455$ & $\underline{0.413 / 0.636}$ & $\underline{7.4 \times 10^{-1} / 5.5 \times 10^{-1}}$ \\
\hline $\begin{array}{l}\text { SHS after Boolean } \\
\text { remission }\end{array}$ & $43.1 / 57.1$ & $43.3 / 56.8$ & $\underline{47.6 / 113.7}$ & $\underline{5.0 \times 10^{-2} / 6.0 \times 10^{-2}}$ \\
\hline $\begin{array}{c}\text { QOLS after } \\
\text { Boolean remission }\end{array}$ & $0.839 / 0.818$ & $0.837 / 0.823$ & $\underline{0.836 / 0.761}$ & $\underline{3.2 \times 10^{-1} / 8.6 \times 10^{-2}}$ \\
\hline
\end{tabular}

Abbreviations: CDAI, clinical disease activity score ; SDAI, simplified disease activity score ; G-IBR, a patient group who attained index-based remission criteria at the same time as attaining Boolean remission or earlier ; G-BR_IF, a patient group who attained Boolean remission followed by index-based remission criteria or failed to attain index-based remission criteria ; DAS28-CRP, 28-joints disease activity score with C-reactive protein ; HAQ, Health Assessment Questionnaire Disability Index ; SHS, 
Sharp/van der Heijde Score ; QOLS, quality of life score calculated from EuroQOL 5 dimensions ; PSVAS, pain score measured with visual analog scale ; n.s., not statistically significant.

All statistical significances are set within 5\%.

There were no statistical significance with the DAS28-CRP, because of number of case was too small in $\underline{\text { the G-BR_IF }}$

In all columns including columns of p-value, average value in the G-IBR and the G-BR IF group separated with slash are shown.

MTX administration rate, average MTX dose, b-/ts-DMARD administration rate, GCS administration rate, and average GCS dose with respect to each variant of the indexbased remission criterion were shown in Table 3. There was no significant difference between the two groups for any variant.

Table 3: MTX, GVS, and b-/ts-DMARD administration rate and dosage before the first Boolean remission and thereafter in regard with each variant index-based remission criteria

\begin{tabular}{|c|c|c|c|}
\hline & CDAI & SDAI & DAS28-CRP \\
\hline $\begin{array}{l}\text { MTX administration rate before } \\
\text { the first Boolean remission }\end{array}$ & $68.0 \% / 76.9 \%$ & $68.7 \% / 64.7 \%$ & $69.0 \% / 20.0 \%$ \\
\hline $\begin{array}{l}\text { average MTX dose before the first } \\
\text { Boolean remission }\end{array}$ & $8.1 / 8.1$ & $8.2 / 7.1$ & $8.1 / 8.0$ \\
\hline $\begin{array}{l}\text { b-/ts-DMARD administration rate } \\
\text { before the first Boolean remission }\end{array}$ & $17.8 \% / 7.7 \%$ & $17.2 \% / 17.6 \%$ & $17.4 \% / 0.0 \%$ \\
\hline $\begin{array}{l}\text { GCS administration rate before the } \\
\text { first Boolean remission }\end{array}$ & $28.8 \% / 23.1 \%$ & $28.3 \% / 35.2 \%$ & $28.6 \% / 20.0 \%$ \\
\hline $\begin{array}{l}\text { average GCS dose before the first } \\
\text { Boolean remission }\end{array}$ & $3.3 / 2.6$ & $3.3 / 3.0$ & $3.3 / 5.0$ \\
\hline $\begin{array}{l}\text { MTX administration rate after the } \\
\text { first Boolean remission }\end{array}$ & $75.0 \% / 76.9 \%$ & $75.7 \% / 58.8 \%$ & $75.6 \% / 20.0 \%$ \\
\hline $\begin{array}{l}\text { average MTX dose after the first } \\
\text { Boolean remission }\end{array}$ & $8.2 / 8.0$ & $8.2 / 8.4$ & $8.1 / 16.0$ \\
\hline $\begin{array}{l}\text { b-/ts-DMARD administration rate } \\
\text { after the first Boolean remission }\end{array}$ & $19.4 \% / 38.5 \%$ & $20.5 \% / 17.6 \%$ & $20.6 \% / 0.0 \%$ \\
\hline $\begin{array}{l}\text { GCS administration rate after the } \\
\text { first Boolean remission }\end{array}$ & $39.6 \% / 26.9 \%$ & $38.9 \% / 41.1 \%$ & $39.1 \% / 20.0 \%$ \\
\hline $\begin{array}{c}\text { average GCS dose after the first } \\
\text { Boolean remission }\end{array}$ & $3.8 / 3.6$ & $3.9 / 3.6$ & $3.8 / 5.0$ \\
\hline
\end{tabular}

Abbreviations: MTX, methotrexate ; GCS, glucocorticoid steroid ; b-/ts-DMARD, biologic or targeted synthetic disease-modifying anti-rheumatic drug; CDAI, clinical disease activity score ; SDAI, simplified disease activity score ; DAS28-CRP, 28-joints disease activity score with Creactive protein.

In all columns, numbers were shown in the G-IBR (a patient group who attained clinical remission with the index-based criteria at the same time with attaining the Boolean remission or earlier) followed by the G-BR-IF (a patient groups who attained the Boolean remission before attaining clinical remission with variant clinical remission) separated by slash.

Units: $\mathrm{mg} /$ week in MTX dose, and mg/day in GCS dose.

Statistical significance: There is no significant difference between the two groups for any of the variant index-based remission criterions. 
Boolean remission rate in the patients who attained index-based remission was 95.1\%, 95.3\%, and 86.7\% with regard to CDAI, SDAI, and DAS28-CRP, respectively. Failure to attain index-based remission rate in the patients who did not attain Boolean remission was $90.7 \%, 90.7 \%$, and $14.8 \%$ with regard to CDAI, SDAI, and DAS28-CRP, respectively (Table 4 ).

Table 4: Patients distributions according to classification with CDAI, SDAI and DAS28-CRP remission

\begin{tabular}{cccc}
\hline & CDAI & SDAI & DAS28-CRP \\
\hline$\underline{\text { G-IBR }}$ & 225 & 231 & 482 \\
$\underline{\text { G-BR_IF }}$ & $160(122: 38)$ & $154(135: 19)$ & $8(6: 2)$ \\
$\underline{\text { G-IR_BF }}$ & 18 & 18 & 75 \\
$\underline{\text { G-BothF }}$ & 175 & 175 & 13 \\
in total & 418 & 424 & 570 \\
\hline Sensitivity & $95.1 \%$ & $95.3 \%$ & $86.7 \%$ \\
Specificity & $90.7 \%$ & $90.7 \%$ & $14.8 \%$ \\
\hline
\end{tabular}

Abbreviations: CDAI, clinical disease activity index ; SDAI, simplified disease activity index ; DAS28-CRP, 28-joints disease activity score with C-reactive protein ; G-IBR, a group who attained index-based remission criteria at the same time as attaining Boolean remission or earlier ; G-BR IF, a group who attained Boolean remission followed by index-based remission criteria or failed ; G-IR_BF, a group who could not attain Boolean remission despite attaining index-based remission criteria ; G-BothF, a group who failed attaining neither Boolean remission nor index-based remission criteria.

In G-BR_IF, numbers of patients who attained index-based remission criteria but later than attaining Boolean remission and number of patients who could not attain index-based remission criteria separated by colon for each criteria are shown in parentheses.

Sensitivity: number of G-IBR divided by number of the patient who attained remission with each index-based remission criteria.

Specificity: number of G-BothF divided by number of the patient who did not attain remission with each index-based remission criteria.

\section{Discussion}

The greatest advantage of adopting T2T in the treatment of RA is the increased likelihood of a stable clinical course that diminishes inflammatory influences of RA on patients [14]. Systematic treatment that targeted clinical remission facilitates the improvement of patients' activity in daily living and maintenance of the quality of life [2]. For that reason, targeted clinical remission should guarantee sustained stable disease activity over a long period. However, the definition of remission in DAS28 had not been validated and may be insufficient to predict a stable clinical course because the remission 
level of DAS28 $\leq 2.6$ still has a severe level of inflammation and, therefore, leads to erosive deformation on the joints $[4,8]$. Therefore, a more stringent index for remission is needed.

The definition of Boolean remission nowadays is the most reliable and sensitive composite measure that can predict sustainable tight disease control and maintenance of the radiographic joint structure. However, even patients who attained Boolean remission develop flare of disease activity and unstable PGA later in real clinical scenarios. Therefore, Boolean remission may be suggested dispersion in the clinical course after remission [7].

We adopted CDAI as an initial target to achieve remission. The main reason for its adoption is that we were afraid of confounding other inflammatory changes in mixing with CRP because CRP is a serological biomarker, and it often reflects infection, interstitial lung reaction, or other inflammation. All other indices such as SDAI, DAS28, and Boolean include CRP as a component. Therefore, these indices may be sensed by other causes of inflammation different from RA itself. However, after observation of the results in the study, SDAI that includes CRP in the index overlaps with CDAI mostly. These results suggest that CRP also includes the inflammation status of RA.

The definition of remission in terms of CDAI is 2.8 or less, and that in terms of SDAI is 3.3 or less. These criteria enable the elevation of PGA up to some level, at most $2.0 \mathrm{~cm}$. Recent studies reported that a PGA of up to $2.0 \mathrm{~cm}$ or $2.5 \mathrm{~cm}$ is allowed at a reliable level to maintain a good clinical course for patients with RA [7,15). If PGA $\leq 2.0 \mathrm{~cm}$ within CDAI or SDAI remission criteria is reliable for the prediction of disease activity prognosis, CDAI and SDAI remission should be equivalent with Boolean remission or near-miss with PGA $\leq 2.0 \mathrm{~cm}[16]$.

One novel advantage of aiming index-based remission such as CDAI and SDAI for the initial target is that a stable and sustainable clinical course after remission is predicted. The results of the study suggest that every clinical component that configures indices such as CDAI and SDAI is superior in the G-IBR group than in the G-BR IF group regardless of CDAI or SDAI, even if these patients attained Boolean remission afterward. Particularly, the average value of DAS28-CRP in the G-BR IF group after Boolean remission was within remission in the index-based remission of DAS28-CRP. However, that value cannot guarantee radiographic maintenance in the joint structure [13]. Results in this study also showed that clinical remission with DAS28-CRP differs significantly from remission with the other two remission criterions in patient distribution. Therefore, targeting CDAI or SDAI remission as an initial goal is a reasonable aim setting.

One more thing that should be emphasized is the high sensitivity for attaining Boolean remission. More than $90 \%$ of patients who attained CDAI or SDAI remission prior to attaining Boolean remission can attain Boolean remission afterward, despite the fact that approximately $60 \%$ of the patients who could not attain CDAI or SDAI remission first cannot attain Boolean remission, too. These results suggest that CDAI or SDAI remission as an initial target is comparable with aiming Boolean remission.

On the other hand, DAS28-CRP demonstrated similar sensitivity and specificity of Boolean remission achievement when DAS28-CRP was achieved as an index-based remission; however, the Boolean failure rate of the patients who failed to achieve DAS28CRP remission was only $13.8 \%$, while this value with regard to CDAI and SDAI was $7.13 \%$ for both index-based remissions. These results show a similarity in the component setting of DAS28-CRP and Boolean setting. However, DAS28-CRP is looser than the 
Boolean setting. Thus, the lower failure rate in the Boolean failure patients in terms of DAS28-CRP than in terms of CDAI and SDAI was demonstrated. These results suggested that DAS28-CRP is not sufficient for the initial target of index-based remission.

One concern remains that even if CDAI or SDAI remission is achieved prior to attaining Boolean remission, the HAQ score, SHS and QOLS demonstrated no significant difference between the G-IBR and the G-BR IF groups. One reasonable explanation might be suggested that these values are not compared with their change but compared with the absolute value. The background clinical characteristics of the G-IBR and GBR IF groups demonstrated no significant differences; therefore, it may be difficult to demonstrate a significant difference between the two groups after Boolean remission, given that all these values are influenced by the values at baseline of each.

The limitations of the current study include that it is a single center study, that it does not consider race, age, gender, etc., and that the target set in the baseline is a single arm. In particular, when Boolean remission is set to primary target, it is difficult to infer the effect of the change of PGA because the result is unknown. Whether the optimal level of the targeted PGA affects disease activity after transmission is the most noteworthy issue, and we can only expect further studies.

\section{Conclusions}

The use of index-based remission criteria as an initial target to attain in accordance with T2T is reasonable and attaining CDAI or SDAI remission at the same time as attaining Boolean remission or earlier suggests the prediction of a stable and settled disease status after attaining Boolean remission.

Acknowledgement: Author appreciated Ms. Kaoru Kuwabara, Sayori Masuoka, Eri Morichika, and Aoi Yoshida for dedicated data collecting. Author also appreciate Ms. Saori Tamura for identical X-ray picture techniques.

Author contribution: Conceptualization, IY; Methodology, IY and NS; Software, IY; Validation, IY, TC, and NS; Formal Analysis, IY; Investigation, IY; Resources, IY, TC, and NS; Data Curation, IY; Writing - Original Draft Preparation, IY; Writing - Review \& Editing, IY, TC, and NS; Visualization, IY; Supervision, TC, and NS; Project Administration, IY; Funding Acquisition, none.

Funding disclosure: No funding received.

Institutional Review Board Statement: This study was approved by Yoshii Hospital Ethics Committee (approval number: Y-RA-2020-2) in accordance with the ethical standards laid down in the 1964 Declaration of Helsinki and its later amendments.

Informed Consent Statement: Anonymity was ensured for all patients and families who participated in this study, and no names and/or addresses were issued that could help identify these individuals.

Conflict of Interest Statement: None of author and his families have share income, property with any person, or any grants or other financial supports of the study. 


\section{References}

1) Smolen JS, Aletaha D, Bijlsma JWJ, Breedveld FC, Boumpas D, Burmester G, et al, for the T2T Expert Committee. Treating rheumatoid arthritis to target: recommendations of an international task force. Ann Rheum Dis 2010;69:631-7.

2) Smolen JS, Landewé RBM, Bijlsma JWJ, Burmester G, Chatzidionysiou K, Dougados M, et al. EULAR recommendations for the management of rheumatoid arthritis with synthetic and biological disease-modifying antirheumatic drugs: 2016 update. Ann Rheum Dis 2017;76:960-77.

3) Fransen J, Creemers MCW, van Riel PLCM. Remission in rheumatoid arthritis: agreement of the disease activity score (DAS28) with the ARA preliminary remission criteria. Rheumatol 2004;43:1252-5.

4) Aletaha D, Smolen JS. The simplified disease activity index (SDAI) and the clinical disease activity index (CDAI): A review of their usefulness and validity in rheumatoid arthritis. Clin Exp Rheumatol 2005;23(5 Suppl 39):S100-8.

5) Felson DT, Smolen JS, Wells G, Zhang B, van Tuyl LHD, Funovits J, et al. American College of Rheumatology/European League against Rheumatism provisional definition of remission in rheumatoid arthritis for clinical trials. Arthritis Rheumatism 2011;63:573-86.

6) Mack ME, Hsia E, Aletaha D. Comparative assessment of the different American College of Rheumatology/European League Against Rheumatism remission definitions for rheumatoid arthritis for their use as clinical trial end points. Arthritis Rheumatol 2017;69:518-28.

7) Studenic P, Felson D, de Wit M, Alasti F, Stamm TA, Smolen JS, Aletaha D. Testing different thresholds for patient global assessment in defining remission for rheumatoid arthritis: are the current ACR/EULAR Boolean criteria optimal?. Ann Rheum Dis 2020;79:445-52.

8) Aletaha D, Wang X, Zhong S, Florentinus S, Monastiriakos K, Smolen JS. Differences in disease activity measures in patients with rheumatoid arthritis who achieved DAS, SDAI, or CDAI remission but not Boolean remission. Seminars Arthritis Rheumatism 2020;50:276-84.

9) Bruce B, Fries JF. The Stanford Health Assessment Questionnaire: Dimensions and practical applications. Health Quality Life Outcome 2003; 1:20.

10) Carlsson AM. Assessment of chronic pain. I. Aspects of the reliability and validity of the visual analogue scale. Pain 1983;16;87-101.

11) Herdman M, Gudex C, Lloyd A, Janssen MF, Kind P, Parkin D, et al. Development and preliminary testing of the new five-level version of EQ-5D (EQ-5D-5L). Quality Life Res 2011;20:1727-36.

12) van der Heijde D, Dankert T, Nielman F, Rau R, Boers M. Reliability and sensitivity to change of a simplification of the Sharp/van der Heijde radiological assessment in rheumatoid arthritis. Rheumatol 1999;38:941-7.

13) Shiroiwa T, Fukuda T, Ikeda $S$, Igarashi A, Noto $S$, Saito $S$, et al. Japanese populating norms for preference-based measures: EQ-5D-3L, EQ-5D-5L, and SF-6D. Qual Life Res 2016;25:707-19.

14) Smolen JS, Landewé RBM, Bijlsma JWJ, Burmester GR, Dougados M, Kerschbaumer A, et al. EULAR recommendations for the management of rheumatoid arthritis with synthetic and biological disease-modifying antirheumatic drugs: 2019 update. Ann Rheum Dis 2020; 79:685-99. 
15) Studenic P, Stamm T, Smolen JS, Aletaha D. Reliability of patient-reported outcomes in rheumatoid arthritis patients: an observational prospective study. Rheumatol 2016;55:41-8.

16) Studenic P, Smolen JS, Aletaha D. Near misses of ACR/EULAR criteria for remission: effects of patient global assessment in Boolean and index-based definitions. Ann Rheum Dis 2012;71:1702-5. 